\title{
Appendix gap losses in Stirling engines - review of recent findings
}

\author{
Hans-Detlev Kühl*, and Jan Sauer \\ TU Dortmund University, Chair of Thermodynamics, Emil-Figge-Str. 70, 44227 Dortmund, Germany
}

\begin{abstract}
The appendix gap loss in Stirling cycle machines is generated by the annular gap around the thermally insulating, thin-walled dome typically attached to a piston or displacer plunging into the hot cylinder volume of an engine or the cold volume of a cryocooler. It was considered to be of minor importance for decades. Thus, simplified analytical models were considered sufficiently accurate for its description, until numerical simulations and experimental results gave rise to a more detailed analysis revealing that, neglecting entrance and end effects, the flow is typically laminar, but unsteady. Subsequently, an enhanced analytical model accounting for fluidic and thermal inertia effects as well as the volumetric displacement by the seal was developed. Compared to the previous ones, this model predicts a shift of the optimum width to smaller values, a higher minimum overall loss and furthermore, an option to decrease the loss by reducing the effective seal diameter. This could be experimentally confirmed as well as the unsteady gas temperature profiles predicted by this model. Subsequently, both theoretically and experimentally founded correlations for the radial and axial energy transport in the gap were derived and implemented in a differential simulation of the gap within a third order code.
\end{abstract}

\section{Introduction}

Stirling engines and similar regenerative cycles are well-suited for the utilization of renewable energy and may therefore be profitably deployed in the current transition to a sustainable energy supply. So, optimization of their performance by an accurate modelling of the various loss mechanisms including their mutual interactions is an ongoing issue.

One of these losses is the so-called appendix gap loss. It is generated in the annular gap between the cylinder wall and the thermally insulating dome or "hot cap" that is typically attached to a piston or displacer plunging into a "hot" (or - in case of a cryocooler - possibly a "cold") cylinder to reduce thermal conduction losses and to protect the seal against high (or cryogenic) temperatures. The gap therefore features a "dead end" at the opposite, typically moderate, near-ambient temperature, since this is of course the preferred location of the seal, and this is presumably the mental image behind the term "appendix gap". The appendix gap loss may be subdivided into several mechanisms according to currently available theories.

\footnotetext{
* Corresponding author: hans-detlev.kuehl@tu-dortmund.de
} 
Leakage losses, i.e., a net enthalpy transfer by an oscillating gas flow past the seal, can generally be minimized by a good seal design, but may possibly be an issue in case of the hot piston of an $\alpha$-Stirling engine and - of course - in case of the close tolerance seals typically used in free piston machines. They are usually not considered to be part of the appendix gap losses, but there will be an interaction with these, if they should be significant. However, in case of the displacer seals in a kinematic $\beta$ - or $\gamma$-Stirling engine or another regenerative machine, e. g. a Vuilleumier heat pump, they are mostly negligible due to the small pressure difference across the seal. This case, i.e., the appendix gap of a double-acting displacer with a regular seal sliding on the cylinder liner, will be exemplarily assumed in the following, since this is the only case that can be generalized without knowledge of design details.

The so-called shuttle loss was probably first observed and described as "motional heat transfer" in the field of cryocoolers [1] and later also referred to as "bucket brigade loss" [2]. It can actually be interpreted as a net energy transport down the temperature gradient via the solid material of the moving dome, since its wall surface takes up heat from a warmer section of the cylinder wall, when the displacer is close to its top dead center (TDC) position, and rejects it to a colder section, when it is near the bottom dead center (BDC). However, the periodic heating and cooling of the displacer wall surface is typically in the range of a few tenths of a Kelvin only. Therefore, the easiest way to describe this loss is by considering the heat exchange between the two interacting walls, which is mainly limited by the insulating gas layer in the gap. This was first done by Rios [3], who also considered the effect of the limited thermal penetration depth in the wall surfaces and the corresponding wall temperature oscillations. However, these usually turn out to be negligible in this context, except for cryocoolers with displacers made from polymers instead of metallic materials. With this simplification, Rios derived an analytical equation for the shuttle loss $\dot{Q}_{s h}$, which is only based on the modelling of the aforementioned heat transfer across the gap by steady-state thermal conduction in the gas, and thus is inversely dependent on the gap width $h$ [4]:

$$
\dot{Q}_{s h}=\frac{\pi}{2} \frac{\hat{x}^{2} d_{C}}{h} \lambda_{\text {gas }} Y
$$

The displacer motion is assumed to be sinusoidal here with an amplitude $\hat{x}$. As is to be expected, the loss is proportional to the cylinder diameter $d_{C}$, the thermal conductivity of the gas $\lambda_{\text {gas }}$ and the temperature gradient $Y=\left(T_{h}-T_{c}\right) / l$, which is assumed to be constant and thus only depends on the hot and the cold temperature, $T_{h}$ and $T_{c}$, and the gap length $l$. In the subsequent years, several authors [5-7] published similar approaches applying slightly deviating assumptions such as a non-sinusoidal displacer motion, which all feature the same dependencies on the aforementioned variables, but with different prefactors other than $\pi / 2$.

The second major mechanism contributing to the appendix gap loss is the so-called enthalpy loss, which is sometimes also referred to as "pumping loss" [8] and is caused by the oscillating gas flow across a given boundary at the open end of the gap, which may either be assumed to be fixed to the cylinder wall or to move with the upper "edge" of the displacer dome. Such an oscillating flow will even occur in the latter case due to density changes caused by fluctuations of the cycle pressure and the average gas temperature in the gap, although the volume of the gap below the boundary is then constant in the case of a regular seal moving with the displacer and sliding on the cylinder wall surface. If a boundary fixed to the cylinder wall is considered, the resulting gas flow is of course essentially dominated by the corresponding change of the gap volume, although fluctuations of the pressure and the average gas temperature may exert additional effects. In any case, the temperature of the gas and thus its specific enthalpy will be dependent on the direction of the flow. Assuming the exemplary case of a gap that is open to the hot cylinder volume of a $\beta$ - or $\gamma$-Stirling engine in the following, the temperature of the gas entering the gap from the cylinder volume will be higher than that of the exiting gas, which emanates from "colder" sections of the gap volume. Therefore, the result will be a net enthalpy flow into the gap in this case. 
As far as known, the first quantitative approach to describe this loss mechanism was contributed by Magee and Doering [5], who treated the gap similarly to a regenerative annulus featuring a reheat loss due to heat transfer limitations. Applying an empirical heat transfer correlation for laminar flow in steam-heated tubes, they obtained a formula for the enthalpy flow loss $\dot{Q}_{e n}$ featuring a dependency on the gap width with an exponent of 2.6 .

A more well-known and widely applied analytical model for the enthalpy loss was later developed by Berchowitz [9] on the basis of his earlier work [8,10]. Assuming a boundary fixed to the cylinder wall at the position of the upper end of the cylindrical displacer dome in its BDC position, a constant temperature gradient $Y$ and thus an average gas temperature given by the logarithmic mean $\bar{T}_{l n}=\left(T_{h}-T_{c}\right) / \ln \left(T_{h} / T_{c}\right)$, he obtains the enthalpy loss as

$$
\dot{H}_{e n}=n \frac{\bar{p} \bar{V}}{R \bar{T}_{l n}} \cdot r c_{p} \hat{x} Y\left(1-\frac{\pi}{4} \cos \theta_{m}\right)
$$

In this equation, the amplitude of the mass flow is expressed as a product of the rotational speed, the average mass as a function of $\bar{T}_{l n}$, the average cycle pressure $\bar{p}$ and the average volume of the gap $\bar{V} \approx \pi d_{C} h l$ according to the ideal gas law, and a dimensionless amplitude

$$
r=\sqrt{\left(r_{x}-r_{T}-r_{p} \sin \theta_{p}\right)^{2}+\left(r_{p} \cos \theta_{p}\right)^{2}}
$$

which is essentially caused by the change of the volume as expressed by the dimensionless stroke amplitude $r_{x}=\hat{x} / l$, but also includes the effects of pressure and temperature changes, as expressed by the dimensionless amplitude $r_{p}=\hat{p} / \bar{p}$ of the pressure and the phase angle $\theta_{p}$, by which it leads the displacer velocity, and the dimensionless amplitude of the local gas temperature in the gap, $r_{T}=\hat{x} Y / 2 \bar{T}_{l n}$, which is locally constant under these assumptions.

The phase angle of the mass fluctuation relative to the displacer stroke can be obtained as

$$
\theta_{m}=\arctan \frac{r_{x}-r_{T}-r_{p} \sin \theta_{p}}{-r_{p} \cos \theta_{p}}
$$

Contrarily to the shuttle loss, the enthalpy loss is thus linearly dependent on the gap width. Assuming that any further loss mechanisms, e. g. the hysteresis loss, are negligible in most cases [10], it is thus easy to determine an optimum width $h_{\text {opt }}$, where the sum of these losses is minimum. $h_{\text {opt }}$ is essentially dependent on $\bar{p}, n$ and $\bar{T}_{l n}$ as well as on the properties of the gas, but almost independent of the stroke, since the value of $r$ according to eq. (3) is largely dominated by $\hat{x}$ and thus, $\dot{H}_{e n}$ features almost the same square dependency on $\hat{x}$ as $\dot{Q}_{s h}$.

Evaluation of the appendix gap loss at $h_{\text {opt }}$ according to these equations usually yields comparatively low values, and so it was common practice in the subsequent decades to apply this theory, which actually is a decoupled, so-called "second order" approach, even in onedimensional differential, so-called "third order" simulations of regenerative machines, until in 2005, Andersen et al. [11] first presented a third order simulation model that also included a differential simulation of the appendix gap. This model yielded far higher values for the appendix gap loss than the aforementioned second order model, and furthermore, the results were found to be severely dependent on the empirical modelling of the heat transfer.

A few years later, Geue et al. [12] found systematic deviations between third order model predictions and experimental results obtained from extensive performance tests conducted with a laboratory-scale machine that may be toggled between different thermodynamic cycles including the Stirling and the Vuilleumier cycle. Thus, the amplitude and the phase angle of the pressure could be varied independently and in addition to variations of the mean pressure, the speed and the operating temperatures. Analyzing the dependencies of the observed deviations on the various parameters, it was found that they largely coincided with those of the enthalpy loss according to eq. (2). This gave rise to the development of a refined analytical model as well as to numerical and experimental investigations of the appendix gap loss and finally to the suggestion of a modified seal and gap design and the derivation of analytical equations for its optimization. This work will be briefly summarized in the following. 


\section{Enhanced Analytical Modelling of Appendix Gap Losses}

The aforementioned models for the appendix gap loss imply the assumption of a linear temperature profile in the axial direction, which is identical for the cylinder and the displacer wall in mid-stroke position of the latter. During the oscillating displacer motion, an alternating radial temperature difference between these profiles is consequently generated, which is also axially constant. Furthermore, it is assumed that the radial temperature profile in the gas between the two opposed walls is also linear, which in turn requires the assumption of plain laminar shear flow and thus an average flow velocity of the gas amounting to half the displacer velocity. Assuming a common seal design with a seal that is attached to the displacer and slides on the cylinder wall surface, the volumetric displacement of this seal is thus double the volume flow under shear flow conditions. Besides this contradiction, another question is whether the flow can actually be considered as laminar and stationary.

To answer these questions within a review of the existing models, Pfeiffer and Kühl [13] applied a simplification of the model for the enthalpy flow loss according to eq. (2), which is obtained by neglecting the effect of pressure changes in the evaluation of $r$ according to eq. (3). As suggested by Kühl [14], the major dependencies in the result for the optimum width may thus be visualized more clearly, and since $r$ essentially depends on $r_{x}$ in most cases, this simplification is particularly acceptable for cycles with a low pressure amplitude.

Furthermore, it was found that the actual amplitude of $\bar{T}_{l n}$ is not the same as that of a local gas temperature at a given position relative to the cylinder wall as quantified by $r_{T}$ in eq. (3). If the upper boundary of the gap volume is assumed as fixed to the cylinder wall, whereas the lower boundary is moving with the seal, one of the corresponding temperatures is rising and the other is falling as the displacer moves, and therefore, the logarithmic mean is only marginally affected. So, $r_{T}$ should rather be omitted in eq. (3), thus giving an additional impetus for the neglect of $r_{p}$. With these modifications, the optimum width is obtained as

$$
h_{\text {opt }}=\sqrt{\frac{R}{c_{p}} \frac{\lambda_{g a s} \bar{T}_{l n}}{2 n \bar{p}}}
$$

thus confirming the aforementioned insignificance of the dependence on the stroke. Applying this result in the evaluation of the Valensi number as a criterion for the impact of inertia effects in unsteady flow, Pfeiffer and Kühl [15] obtained the simple, constant result

$$
R e_{\omega, o p t}=\frac{\left(2 h_{o p t}\right)^{2} \omega}{v}=\frac{4 h_{o p t}^{2} \omega \bar{p}}{\eta R \bar{T}_{l n}}=\frac{4 \pi}{P r}=6 \pi
$$

for an ideal gas with the theoretical value $\operatorname{Pr}=2 / 3$ of the Prandtl number and the angular velocity $\omega=2 \pi n$. This clearly indicates that the flow in a gap optimized according to the above theory must be considered as unsteady, i.e., inertia effects should be accounted for.

To answer the question whether the flow is laminar, an estimate for the displacer stroke may be obtained by minimizing the sum of thermal conduction and appendix gap losses for a gap optimized according to eq. (5) and under the assumption that the required cylinder wall thickness is given by Barlow's formula $[14,15]$. Thus, the Reynolds number is obtained as

$$
R e_{\text {opt }}=\frac{2 h_{\text {opt }} \hat{u}_{m}}{v}=\frac{4 \pi}{\operatorname{Pr}}\left[\frac{1}{144 \pi}\left(\frac{\lambda_{C}}{\lambda_{\text {gas }}} \frac{\bar{p}}{\sigma_{\text {allow }}}\right)^{2} \frac{V_{C}}{h_{o p t}^{3}}\right]^{1 / 5}
$$

with $\hat{u}_{m}=\hat{x} \omega$ denoting the amplitude of the displacer velocity and thus - ignoring density changes - of the cross sectional average of the flow velocity in the gap, whereas $\lambda_{C}$ and $\sigma_{\text {allow }}$ denote the thermal conductivity and allowable strength of the cylinder wall, respectively, and $V_{C}$ the swept volume of the cylinder. Evaluation of eq. (7) for typical values of these yields $R e_{\text {opt }}<10^{3}$, and due to the small exponent of $1 / 5$ only, even substantial deviations only have a marginal effect on the result. So, the flow can generally be considered as laminar. 
Therefore, it is possible to solve the Navier-Stokes equation analytically under just a few simplifying assumptions, i.e., fully developed flow, a sinusoidal displacer and seal motion and a constant density and viscosity. Subsequently, it is possible to solve the energy equation and thus to derive closed-form solutions for the gas temperature profiles in the gap for a harmonically oscillating, unsteady laminar flow, once again just assuming a fully developed flow and constant fluid properties, i.e. constant values of $\lambda_{g a s}, c_{p}$ and the density $\rho$.

As far as known, the first solution of this problem was provided by Chang et al. [16], who considered a gap that is open at both ends, i.e., the case of a close-tolerance seal as typically found in free piston engines. However, they assume a zero axial pressure gradient and thus obtain a closed-form solution for an unsteady oscillating drag flow without any superimposed pressure-driven component. The resulting profiles of the dimensionless flow velocity $u^{*}=$ $u / \hat{x} \omega$ and the dimensionless temperature $\Theta=\left(T-T_{C W}\right) / Y \hat{x}$ vs. the related radial position $y / h$ are shown in Fig. 1 for $R e_{\omega}=35$ and $\operatorname{Pr}=2 / 3$ at various crank angles $\phi=\omega t$.
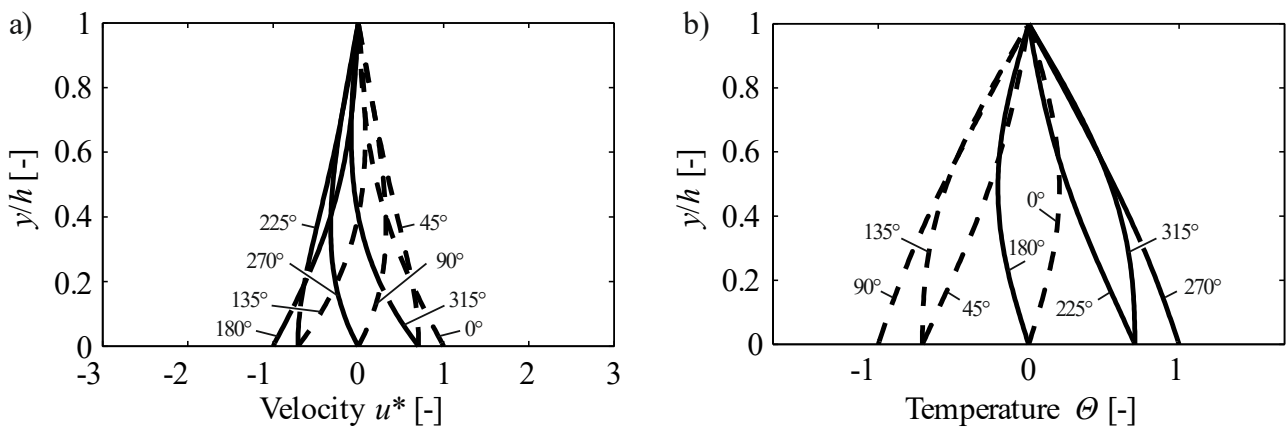

Fig. 1: Dimensionless flow velocity $u^{*}$ and gas temperature $\Theta$ for unsteady oscillating drag flow according to Chang et al. [16] $\left(R e_{\omega}=35, \operatorname{Pr}=2 / 3\right.$, depiction according to [15])

$T_{C w}$ denotes the cylinder wall temperature and thus the gas temperature at $y=1$. The curves for $u^{*}$ clearly indicate a phase shift caused by the mass inertia of the fluid, particularly those at $90^{\circ}$ and $270^{\circ}$ crank angle, which should be zero under quasi-steady conditions, i.e. for $R e_{\omega} \rightarrow 0$. Evidently, the spatial mean flow velocity $u_{m}$ of the gas lags the displacer velocity $u_{D}=\hat{x} \omega \cos (\omega t)$. Regarding the curves for $\Theta$, it is most striking that those at $=0^{\circ}$ and $180^{\circ}$ indicate a non-zero heat flux at the displacer wall surface (i.e., at $y=0$ ), although the difference between the displacer wall temperature and the spatial mean gas temperature should be zero under quasi-steady conditions. Instead, the heat flux is almost zero at $135^{\circ}$ and $315^{\circ}$ crank angle. So, there clearly is a phase shift between the heat flux and the displacer position $x_{D}=\hat{x} \sin (\omega t)$, which definitely affects the evaluation of the shuttle loss described in chapter 1. Chang et al. therefore derive a correction factor for the loss according to eq. (1), which depends on $R e_{\omega}$ and also on the thermal penetration depths of the wall materials, similarly to correction factors derived by Baik and Chang [17] and by Radebaugh and Zimmerman [7] before. As mentioned before, these only take effect in the rather special case of polymers as sometimes used in cryocoolers, which will not be further considered here.

Contrarily to Chang et al., Pfeiffer and Kühl [15] solved the aforementioned equation system for the case of a displacer gap that is closed by a seal at the moderately tempered end and subsequently applied it to investigate the effects of several conceivable modifications of the gap and seal design [18]. As illustrated in Fig. 2, one of these is a variation of the gap width by a conical shape of the cylinder wall, which may be quantified by the conicity $c_{h}$ indicating how much the width at the open end exceeds the initial value $h(0)$ at the lower end of the externally adiabatic section of the gap. As a refinement of the above assumptions, the temperature difference $T_{h}-T_{c}$ is assumed to apply to this adiabatic section of length $l_{a d}$ only and that the entire length $l_{a}$ of the appendix gap also comprises isothermal sections $l_{b o t}$ 
and $l_{\text {top }}$ at the ends, which may vary in size as the displacer moves. Furthermore, the gap width in the bottom section of the gap, i.e., at negative values of the related axial coordinate $x^{*}=x / l_{a d}$, may be reduced to $h_{b o t}<h(0)$ as an additional design option. Thus, it is possible to reduce the volumetric displacement by the seal, possibly even down to zero.

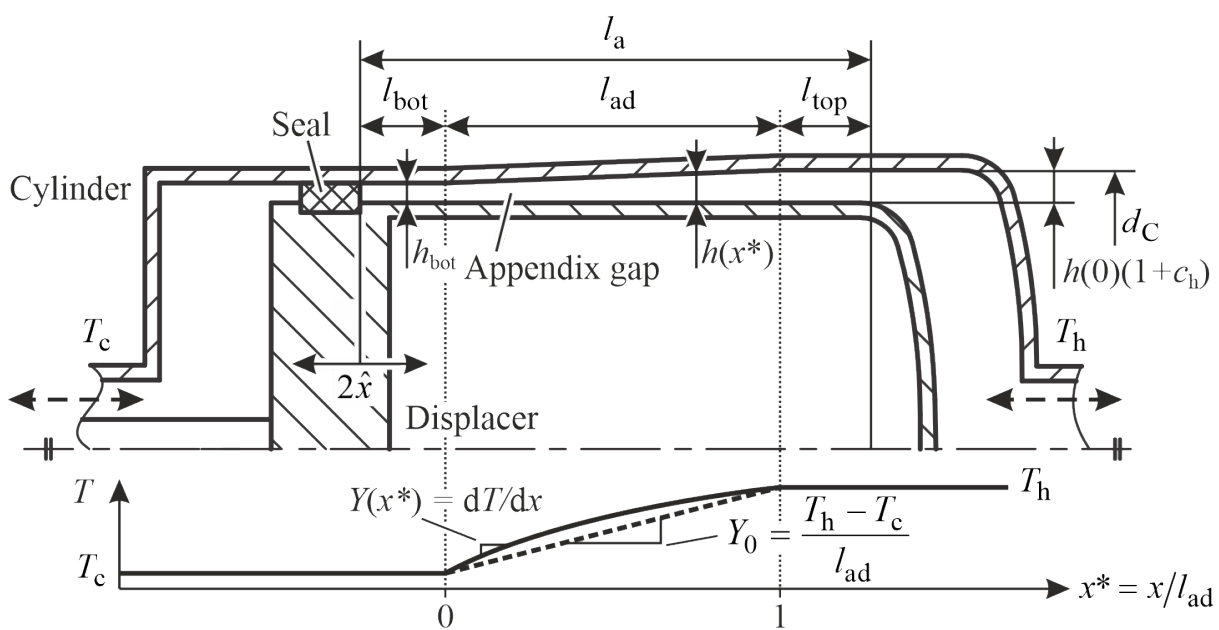

Fig. 2: Cross section of a cylinder-displacer system, introducing the lengths $l_{a}, l_{a d}, l_{b o t}$ and $l_{\text {top }}$ and including the major options to improve the gap and seal design as quantified by the conicity $c_{h}$ and a deviating bottom gap width $h_{b o t}<h(0)$ (depiction according to [18])

The analytical solutions for $u^{*}$ and $\Theta$ obtained by Pfeiffer and Kühl [15] differ from those according to Chang et al. [16] due to an additional pressure flow component and are complex in both senses of the word. They are not reproduced here for reasons of conciseness, but they depend on the amplitude $\Gamma$ and the phase angle $\theta_{u}$ of the complex spatial mean flow velocity ${ }^{\dagger}$

$$
\frac{\boldsymbol{u}_{m}}{\hat{x} \omega}=\frac{\hat{u}_{m}}{\hat{x} \omega} e^{i\left(\phi-\theta_{u}\right)}=\Gamma e^{i\left(\phi-\theta_{u}\right)}
$$

$\Gamma$ is thus the ratio between the amplitude of the spatial mean flow velocity $\hat{u}_{m}$ at a given position in the gap and that of the displacer velocity. Considering the compressibility of the gas as well as the temperature as a function of $x^{*}$, it is possible to evaluate $\Gamma$ and $\theta_{u}$ at any position $x^{*}$ within the adiabatic section, i.e., for $0<x^{*}<1$, and $\Gamma$ is found to increase towards the open end due to the rising temperature as well as to compression and expansion effects. The latter also cause an increasing phase shift $\theta_{u}$, since the pressure fluctuation will never be in phase with the displacer motion in any regenerative cycle. Thus, it is possible to account for density changes in the section of the gap below $x^{*}$ by an appropriate evaluation of $\Gamma$ and $\theta_{u}$, though the analytical solution of the differential equations requires assuming constant fluid properties including the density. Close to the seal and assuming $h_{b o t}=h(0)$, we thus have $\Gamma=1$ and $\theta_{u}=0$, but if the bottom gap width is reduced to $h_{b o t}=h(0) / 2$, the velocity ratio is also decreased to $\Gamma=0.5$, which corresponds to the spatial mean flow velocity in case of quasi-steady drag flow and therefore is the closest possible approximation to the solution by Chang et al. as displayed in Fig. 1. However, the solutions are still different for $e_{\omega}>0$, since there is a phase shift in the latter, whereas the seal enforces a phase angle $\theta_{u}=0$. This is illustrated in Fig. 3 a1). Evidently, the flow velocity at $90^{\circ}$ and $270^{\circ}$ crank angle lags the displacer velocity near the displacer wall, where inertia forces prevail due to the high velocity level, whereas it leads the displacer velocity near the cylinder wall, thus compensating the lag below and ensuring that the spatial mean velocity is zero. Moreover, this minor deviation results in substantially different temperature profiles in Fig. 3 b1).

\footnotetext{
$\dagger$ Complex quantities are generally designated by bold symbols in the following.
} 


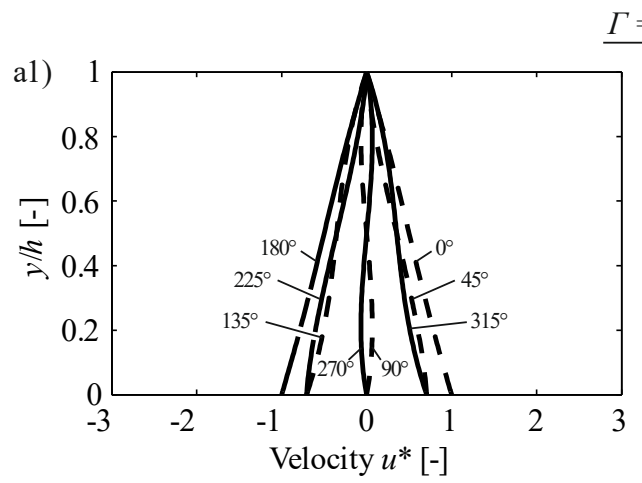

$\Gamma=0.5, \theta_{\mathrm{u}}=0$ :

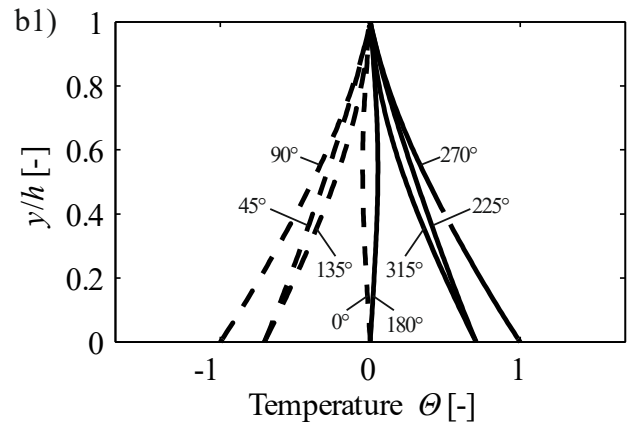

$\Gamma=1, \theta_{\mathrm{u}}=0$ :
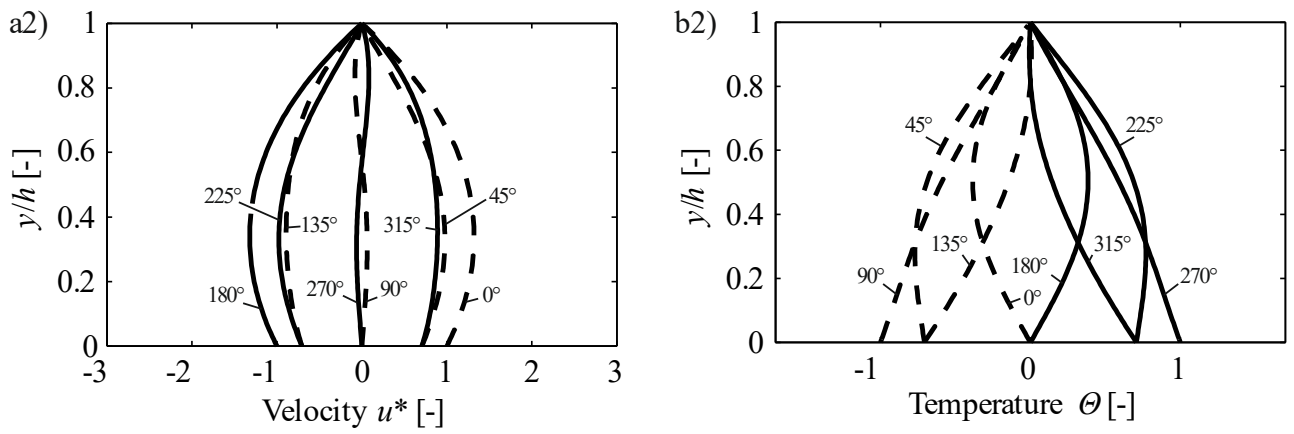

$\underline{\Gamma=2, \theta_{\mathrm{u}}=0:}$
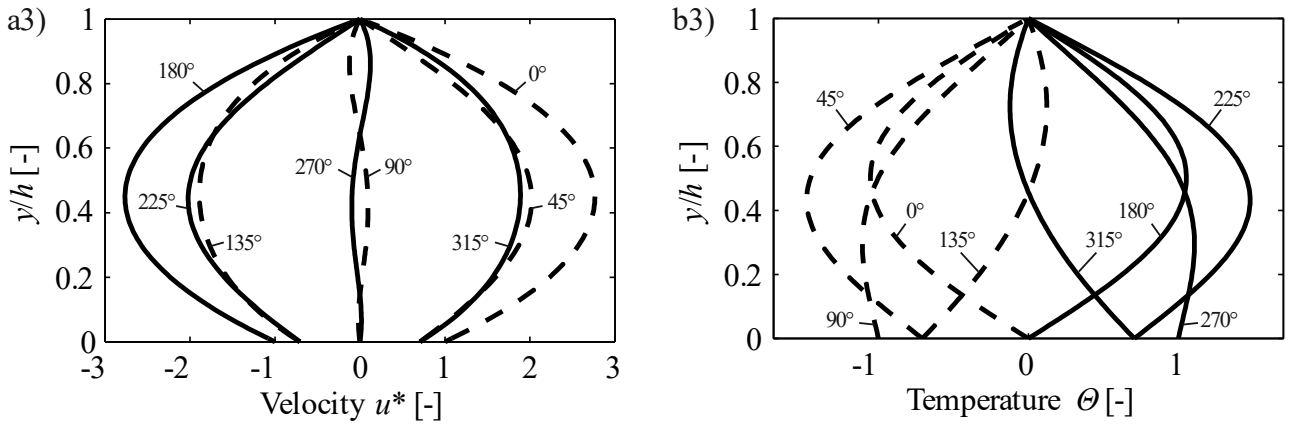

$\frac{\Gamma=2, \theta_{\mathrm{u}}=-\pi / 4:}{\mathrm{b} 4)}$
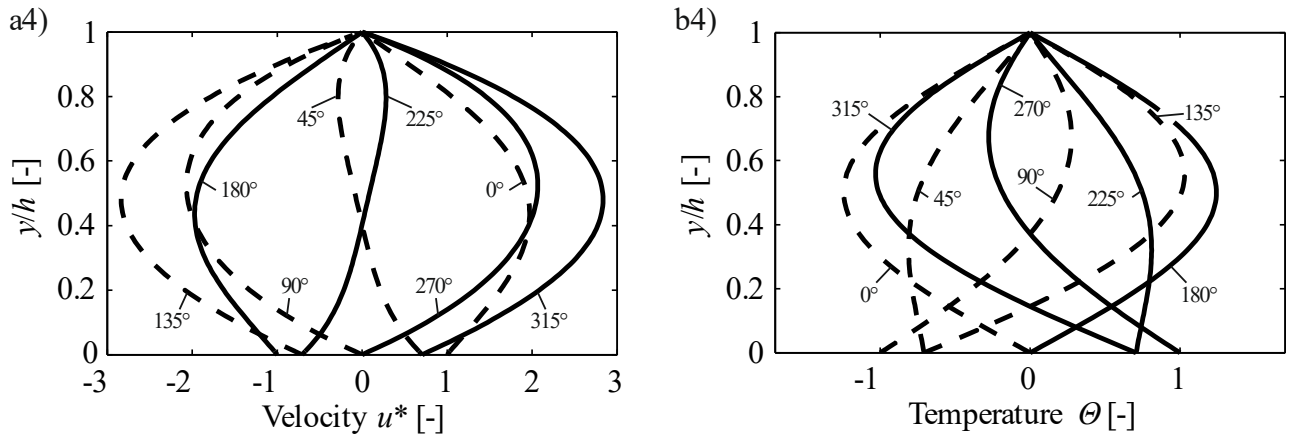

Fig. 3: Dimensionless flow velocity $u^{*}$ and gas temperature $\Theta$ for unsteady oscillating drag flow in a sealed appendix gap for various selected values of the velocity ratio $\Gamma$ and its phase angle $\theta_{u}$ according to Pfeiffer and Kühl [15] $\left(R e_{\omega}=35, \operatorname{Pr}=2 / 3\right)$ 
Evidently, the inclination of the temperature profiles for $0^{\circ}$ and for $180^{\circ}$ is almost vertical at the displacer wall surface, indicating a far smaller magnitude and moreover, an opposite sign for the phase shift of the heat flux compared to the solution by Chang et al., and yielding a deviating result for the shuttle loss, of course. This applies even more to the second case displayed in Fig. 3 a2) and b2), which corresponds to the regular seal design with a bottom gap width $h_{b o t}=h(0)$. The superimposed pressure flow component is clearly detectible from the almost parabolic shape of the velocity profiles in this case. Furthermore, there is a substantial phase shift of the heat flux, the sign of which is still opposite to that in Fig. 1.

Fig. 3 a3), a4), b3) and b4) show solutions for the example case $\Gamma=2$, which is a typical value at a position further away from the seal, possibly even at the open end of gap, depending on the choice of $c_{h}$ and $h_{b o t}$. In case of $\theta_{u}=0$, the pressure flow component as well as the phase shift of the heat flux are further increased, the latter even beyond $90^{\circ}$. However, the case $\theta_{u}=-\pi / 4$ is more realistic here, since the effect of the pressure fluctuation typically generates phase shifts of the spatial mean flow velocity in this range. Evidently, this further affects both the flow velocity and the temperature profiles, indicating that the effect of the phase-shifted pressure oscillation should definitely be accounted for.

On the basis of the analytical solutions, it is subsequently possible to evaluate both the shuttle loss and the enthalpy loss at the open end of the gap as well as at any other position within the adiabatic section. However, the resulting complex equations are not reproduced here for reasons of conciseness. Considering that the center section of the gap is assumed to be externally adiabatic, it is essential to make sure that energy conservation is maintained for the overall sum of all thermal losses, i.e., the shuttle loss, the enthalpy loss and the thermal conduction loss via the cylinder and the displacer wall. Evaluating these losses at different positions along the gap, it was found that any of these is substantially dependent on $x^{*}$, even the latter due to the temperature-dependent thermal conductivity of stainless steel, the most commonly used wall material. As a result, the overall loss increases towards the open end of the gap, if a constant temperature gradient is assumed. Consequently, this assumption was dropped by Pfeiffer and Kühl [18], and a curved temperature profile was derived, the gradient of which increases towards the seal. This finding agrees well with the numerical simulation results obtained by Andersen et al. [11] and was confirmed by experimental investigations, which will be discussed further below. Unfortunately, this profile could only be determined numerically by an iterative shooting method. However, the effects of the aforementioned design options could thus be analyzed extensively, and optimizations minimizing the overall loss could be performed under various constraints. It was found that the overall loss can be substantially reduced by only optimizing the gap width $h(0)$ and the conicity $c_{h}$ without any reduction of the bottom gap width, i.e., if the so-called bottom gap width ratio is maintained at $r_{h}=h_{\text {bot }} / h(0)=1$. However, a comparatively high value $c_{h} \approx 5$ was required in the example case of the well documented GPU-3 Stirling engine $[19,10]$, and an almost identical improvement could be achieved without a conical gap, just by optimizing $h$ at a bottom gap width ratio $r_{h}=0$. When all parameters were optimized simultaneously, only a marginal further improvement was obtained, and the contribution of the minor conicity $c_{h} \approx 0.7$ was so low that the machining effort for its realization is probably not worthwhile. Therefore, Pfeiffer and Kühl concluded that it is most recommendable to avoid a conical gap geometry and rather choose $r_{h}=0$ instead. This may be either realized by a seal attached to the cylinder wall and sliding on the displacer surface, or by a cylinder liner with a diameter just marginally above that of the displacer (which is probably the best solution under manufacturing aspects).

In general, it was observed that the optimum gap widths obtained in these optimizations were roughly just half the value according to eq. (5), and therefore, the Valensi number according to eq. (6) is further reduced to $R e_{\omega} \approx 12$. For such low values, the exact, unsteady solution of the Navier-Stokes equation may be substituted by the well-known parabolic velocity profile obtained for steady-state laminar flow without any major loss of accuracy. 
However, introducing the corresponding simplification in the solution of the energy equation yielded severely deviating results and is therefore not possible. Nevertheless, the complex solution for the temperature profiles could be substantially simplified by the assumption of a parabolic velocity profile, and the same applies to the expressions for the shuttle loss and the enthalpy loss, which are found to depend on the hyperbolic tangent function of a complex quantity $=\sqrt{i R e_{\omega} P r} / 4$, the absolute value of which is below unity under the above conditions. Therefore, the Taylor series expansion of this function converges very fast. Thus, a real-valued polynomial equation is obtained for the optimum kinetic Péclet number $P e_{\omega, \text { opt }}=R e_{\omega, \text { opt }} \operatorname{Pr}$ that may be truncated after the second order term. Its solution is

$$
P e_{\omega, o p t}=\frac{16}{\sqrt{\frac{51}{35}\left[1-4 \Gamma \cos \theta_{p}+4 \Gamma^{2}\right]+\frac{12}{5}\left[2 \Gamma+\sin \theta_{p}\right] f+f^{2} \cos ^{2} \theta_{p}}-f \cos \theta_{p}}
$$

for the special case $r_{h}=0$. It was further found to be sufficiently accurate to evaluate $\Gamma$ and

$$
f=r_{p} \frac{R}{c_{p}} \frac{T_{C w}}{\hat{x} Y}
$$

only once at an intermediate position $x^{*}=0.5$, although they actually are functions of $x^{*}$.

Consequently taking the thermal diffusivity $a=\lambda_{g a s} / \rho c_{p}$ at an average temperature corresponding to $x^{*}=0.5$, the optimum gap width may finally be obtained as follows [18]:

$$
h_{\text {opt }}=\frac{1}{2} \sqrt{\frac{a\left(\left(T_{h}+T_{c}\right) / 2\right)}{\omega} P e_{\omega, o p t}\left(x^{*}=0.5\right)}
$$

\section{Experimental Investigation of the Appendix Gap Loss}

The analytical model for the appendix gap loss presented in the previous chapter is based on the assumption of constant property data of the fluid and of unsteady, oscillating and fully developed laminar flow. Although these assumptions are most likely more realistic than the even more simplifying assumptions made in the earlier models, they are still an idealization of the real situation. Considering the limited overall length of a typical appendix gap, the assumption of developed flow is particularly questionable, since the velocity profile is permanently disturbed by gas from the cylinder volume, which will usually be in a turbulent state and periodically enters the open end of the gap, as well as by the seal and - in case of $r_{h}<1-$ the step in the cylinder wall at the bottom end. Considering that the hydrodynamic entry length in laminar flow may extend to more than 50 times the hydraulic diameter, this assumption will probably apply to a small section around the center of the gap only, if at all. So, Sauer and Kühl performed experimental investigations to verify the predictions by the analytical model [20,21]. For this purpose, an experimental machine was used, the design of which had been downsized to laboratory scale by application of similarity-based scaling rules [22]. It was extensively tested within the research project mentioned in the introduction [12], providing the experimental data that initiated the scrutinization of the existing models for the appendix gap loss. This machine is particularly well suited for such investigations, because it features an enlarged gap width of $h=1.4 \mathrm{~mm}$ due to the scaling procedure, whereas typical gap widths in regular machines are in the range of few tenths of a millimeter. So, there are excellent prerequisites for a direct investigation of the flow and temperature profiles at a high spatial and temporal resolution by inserting appropriate probes directly into the gap. Furthermore, it may be operated either as a Stirling engine or a Vuilleumier heat pump, so that the effects of different pressure amplitudes and phase angles can be studied. Based on experiences with high-speed temperature measurements by fine-wire thermocouples [23,24], it was decided to apply this technique once again. Fig. 4 shows one of the probes that were developed for this purpose. The actual thermocouple features a wire diameter of $12 \mu \mathrm{m}$ only 


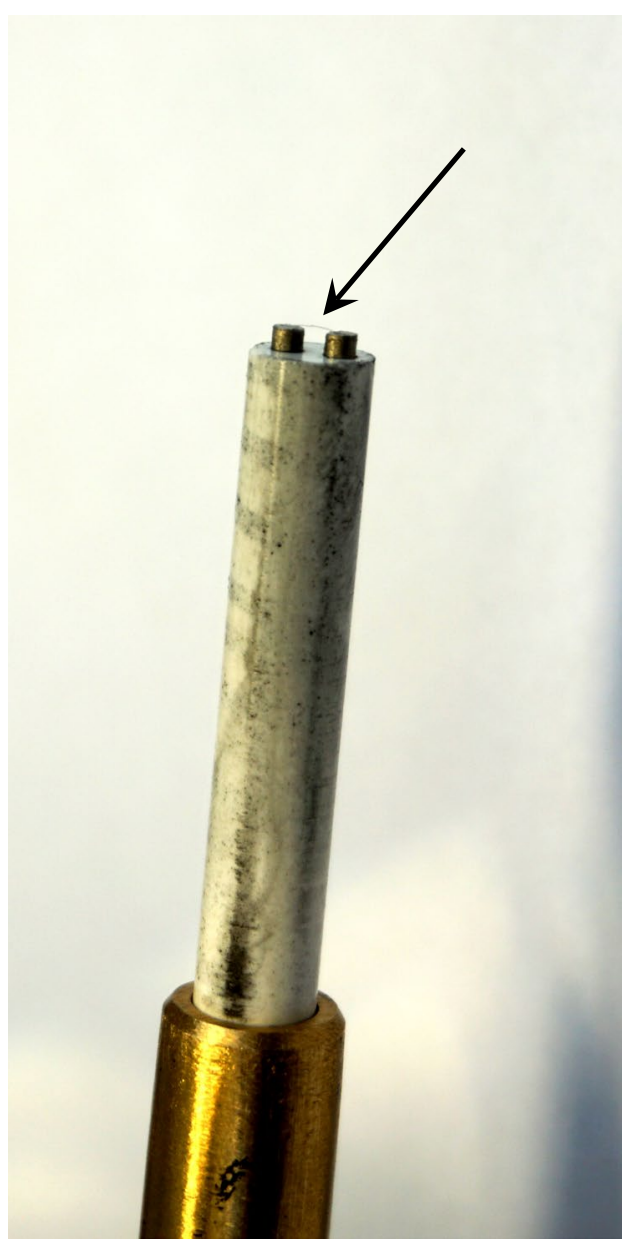

Fig. 4: Photograph of the tip of a fine-wire thermocouple probe deployed to capture gas temperature profiles in the appendix gap

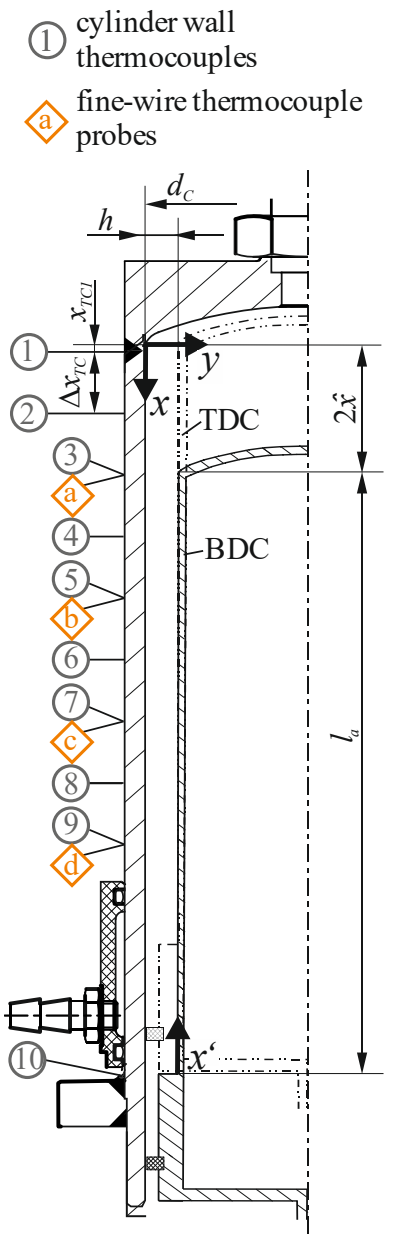

Fig. 5: Schematic cross section of the hot cylinder of the laboratory-scale machine indicating the thermocouple positions [21]

and was spot-welded onto the tips of two solid support wires consisting of the corresponding thermocouple alloys. These are embedded in a ceramic sleeve and may be adjusted axially by a micrometer head. The photograph shows them in their outermost position, in which they would already touch the moving displacer wall. The probes are inserted radially into the gap through bores in the cylinder wall at four different axial positions as indicated in Fig. 5, so that the tips of the ceramic sleeves are level with the inner wall surface. The outer connections used for mounting, positioning and sealing are not shown. The schematic drawing in Fig. 5, which is not to scale (particularly regarding the gap width), also indicates the positions of 9 additional thermocouples that were spot-welded onto the outer wall surface to record the axial wall temperature profile. Contrarily to this illustration, the four probes are not positioned in line but are distributed on different circumferential positions, each $90^{\circ}$ apart from the next, so that mutual disturbances by wakes, which are inevitably caused by any probe, are avoided.

Fig. 6 shows exemplary gas temperature readings obtained in Stirling mode operation. 10 consecutive revolutions have been monitored at each of the given radial positions $y=$ $0.1 \mathrm{~mm}$ through $=1.1 \mathrm{~mm}$. Evidently, the closest similarity to the theoretically predicted sinusoidal curves is found in the center section of the gap, particularly at probe c, although deviations are found even here - presumably caused by the mass flow reversals mainly. 


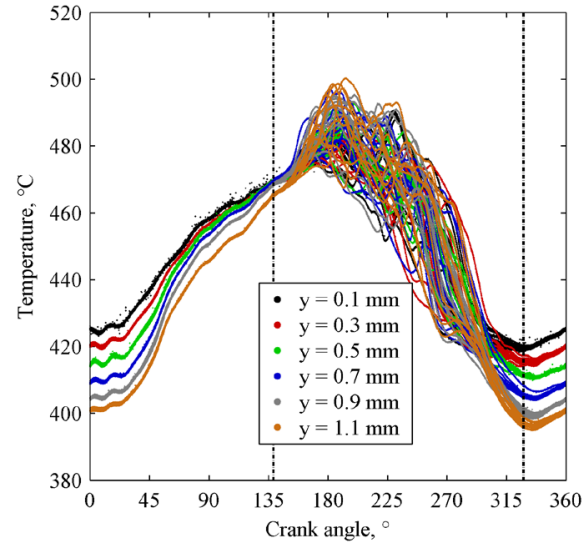

Probe a

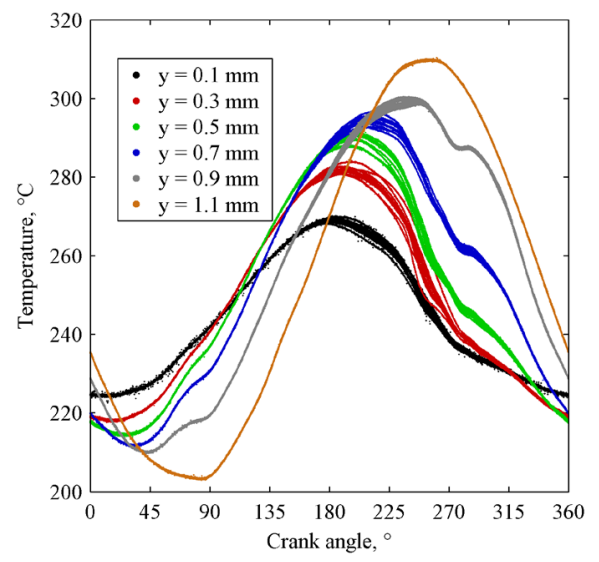

Probe c

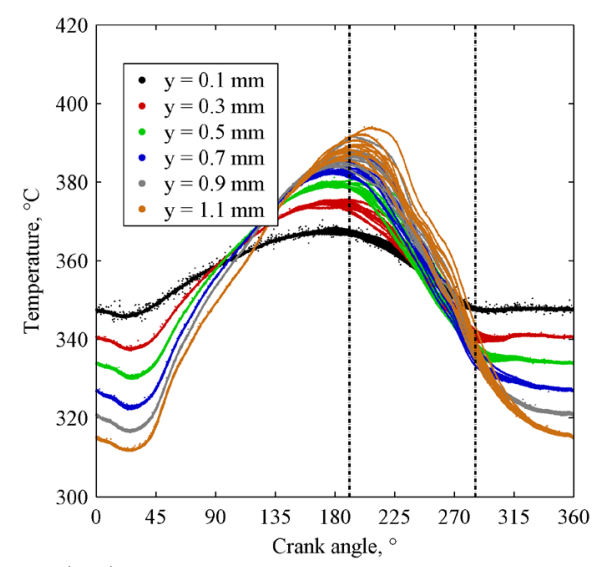

Probe $b$

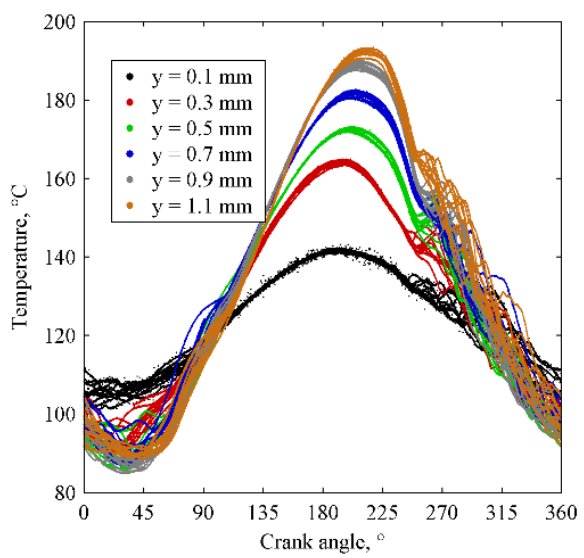

Probe d

Fig. 6: Exemplary gas temperature readings vs. crank angle obtained by the four probes at the positions shown in Fig. 5 in Stirling mode operation of the laboratory-scale machine [21]

The readings obtained from probe $\mathrm{a}-$ and to a lesser extent also those from probe $\mathrm{b}-$ are severely disturbed by turbulent vortices entering the open end of the gap with the inflowing gas from the hot cylinder volume. Considering the velocity ratio $\Gamma$ at this position, it can be demonstrated that these actually penetrate up to the position of probe $b$ approximately [21].

Instead, the disturbances in the readings obtained from probe $\mathrm{d}$ can presumably be explained by the turbulent wake that is generated by the seal on its way downwards along the cylinder liner, which apparently persists for approximately half a cycle and is therefore pushed upwards across the probe after reversal of the displacer motion.

Contrarily to previous experiences, it was further found that the measured temperature profiles required an additional correction regarding both amplitude and phase angle, which was based on a CFD simulation of the periodic gas flow around the thermocouple wire. The reason why this correction was required is that due to the small displacement of the gas in the gap, the amount of gas actually interacting with the probe is so small, that it is inversely influenced by the thermal inertia of the latter. In contrast, previous measurements of unsteady gas temperatures were taken in central flow passages within a machine, where the amount of gas as well as its average flow velocity were so high that this problem did not arise.

Fig. 7 shows the corresponding plots of the corrected gas temperatures vs. the radial position $y$ in the gap and in comparison to the analytically predicted temperature profiles. 


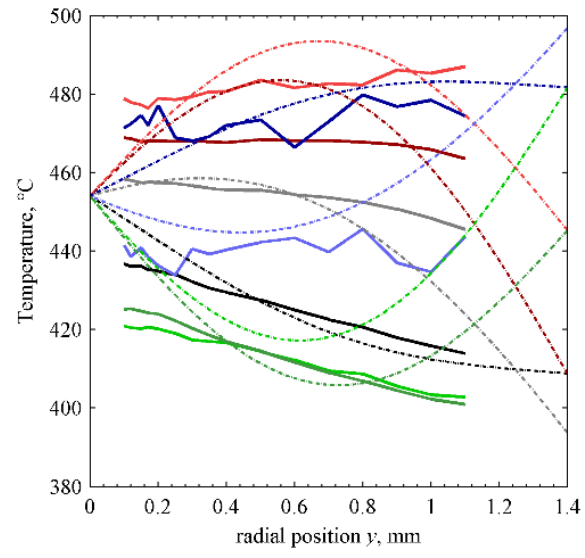

Probe a

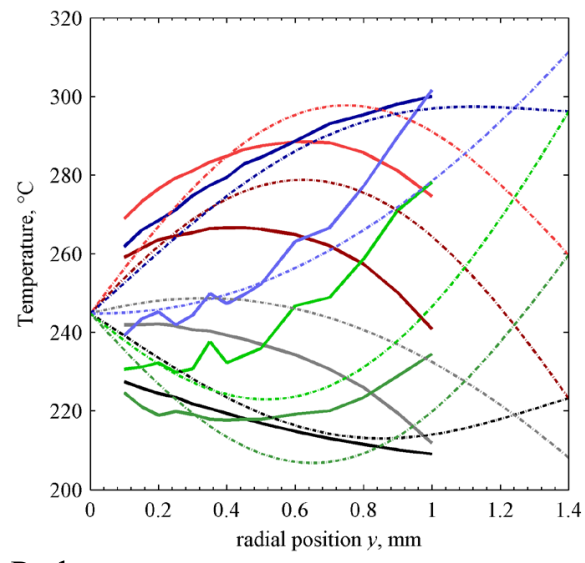

Probe c

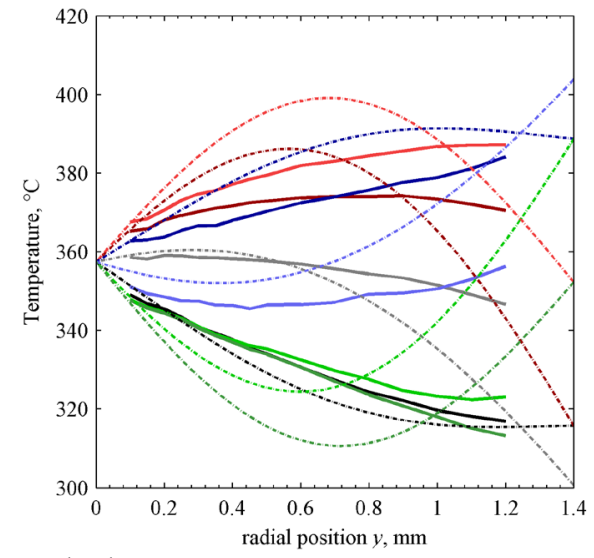

Probe b

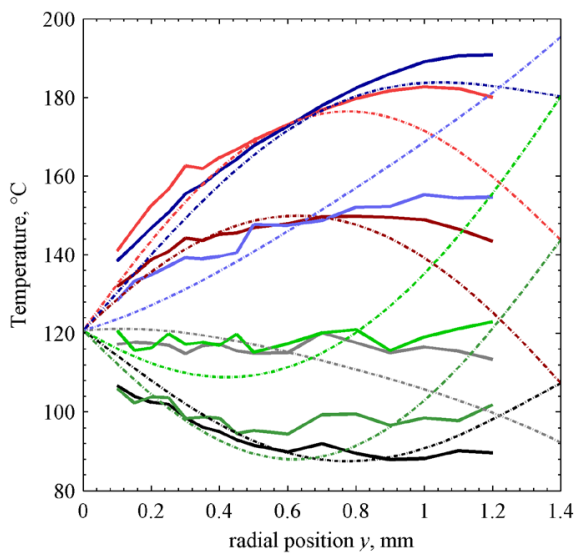

Probe d

$225^{\circ}-315^{\circ}$
$270^{\circ}$
$360^{\circ}$

Fig. 7: Exemplary plots of the corrected experimental gas temperatures vs. the radial position in the gap for the four probe positions according to Fig. 5 in Stirling mode operation of the laboratory-scale machine, in comparison to the analytically predicted curves [21]

Again, the closest similarity is found in the center section of the gap, particularly at the position of probe $\mathrm{c}$, indicating that the assumption of developed flow is best fulfilled here. However, noticeable deviations exist throughout, particularly at the probes where the readings were disturbed by turbulent vortices, of course. The deviations in the center section may be attributed to uncertainties about the temperature profile in the opposed displacer wall, which could not be measured directly. It most likely features an offset relative to the local cylinder wall temperature and moreover, a deviating gradient, both of which could only be estimated. Besides, the assumption of developed flow is most likely not entirely fulfilled anywhere in this gap due to its comparatively large width in relation to a typical length. This actually constitutes a similarity violation that had to be accepted to restrict the dead volume.

Therefore, the assumption of developed flow is presumably better fulfilled in an appendix gap with a regular width of a few tenths of a millimeter only. Despite the deviations, it can thus be concluded that the presented analytical model provides the closest approximation of the actual, evidently very complex situation in the appendix gap that is currently available. 
To additionally verify the aforementioned optimization result concerning the seal design obtained by Pfeiffer and Kühl on the basis of this analytical model, i.e., the recommendation to reduce the gap width as far as possible, the experimental machine was modified once again by reducing the outer diameter of the seal and the displacer base as far as possible and by inserting a new cylinder liner with an accordingly reduced inner diameter [25]. It was not possible to realize a value of zero for the gap width ratio, but the minimum value $r_{h}=0.21$ is very close to the actual, numerically obtained optimum according to Pfeiffer and Kühl [18]. Fig. 8 illustrates this modification in comparison to the original design according to Fig. 2.

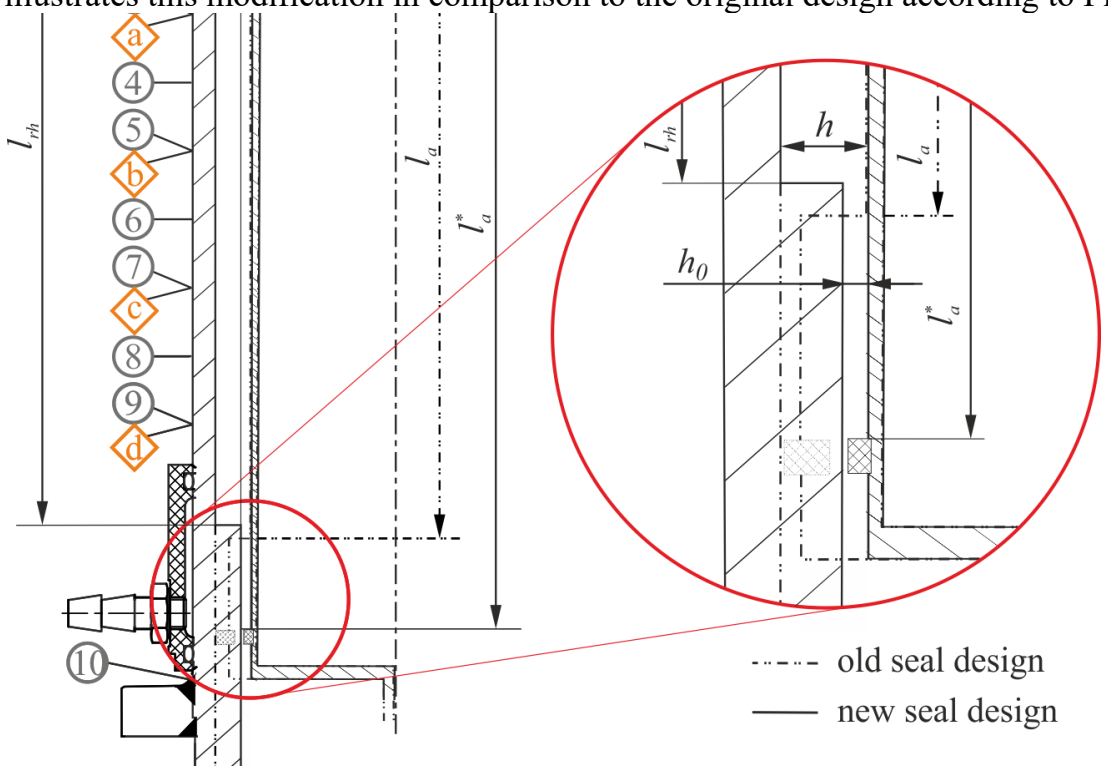

Fig. 8: Illustration of the modification of the seal design as realized by Sauer and Kühl [25]

Considering that the actual volumetric displacement is decreased by this modification, a reduced specific power of the cycle had to be accepted as a side effect, since the closed-cycle $\mathrm{p}, \mathrm{V}$-integrals designating the indicated work and heat exchange of the cycle, respectively, are accordingly diminished by the reduction of the stroke volumes next to the displacer and furthermore, by a decreased amplitude of the pressure due to the lower thermal compression.

Fig. 9 exemplarily shows the $\mathrm{p}, \mathrm{V}$ plots and the wall temperature profiles obtained in Stirling mode under nominal operating conditions as specified in [25]. Surprisingly, it turns out that the pressure amplitude is almost unchanged despite the reduced displacer stroke volume. To some extent, this may be attributed to the slightly reduced dead volume as indicated by the lateral shift of the p,V-plot for the total volume. However, the effect is so substantial that this cannot be the only reason. It can be observed in any of the investigated operating modes and generally results in an improved performance as illustrated in Fig. 10 by a comparison of the externally measured heat flows in either mode as well as the indicated power in Stirling mode. The latter is slightly increased, whereas the hot heat flow is noticeably reduced, i.e., both specific power and efficiency are improved despite the reduced displacer stroke volume. In Vuilleumier mode, a constant refrigeration power is observed, whereas the hot end heat input is once again reduced. The minor change in the axial wall temperature profile displayed in Fig. $9 \mathrm{~b}$ indicates a slightly reduced shuttle loss, but the modification of $r_{h}$ mainly affects the enthalpy loss. Besides, the curved shape of the profile qualitatively confirms the aforementioned numerical results obtained by Andersen et al. [11].

However, the reduction of the appendix gap loss by the modification of the seal design may well explain a reduced hot end heat input to some extent, but is no sufficient explanation for the virtually constant indicated power and refrigeration power, respectively. 


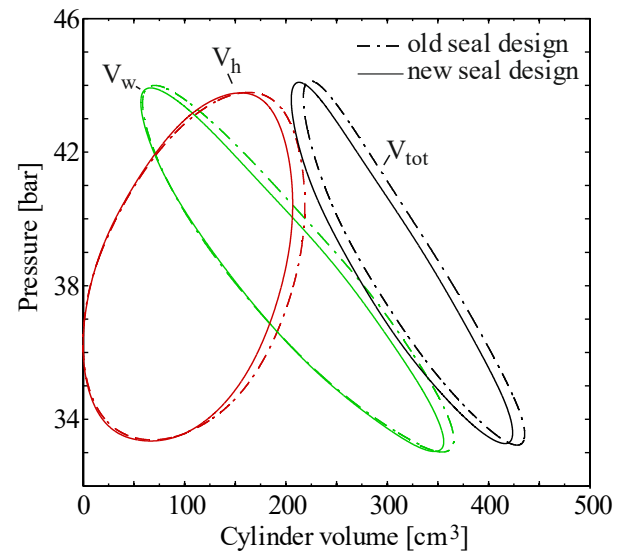

a)

Fig. 9: Experimental p,V-plots and wall temperatures scale machine with old and new seal design at nominal conditions except $\bar{p}=20$ bar [25]

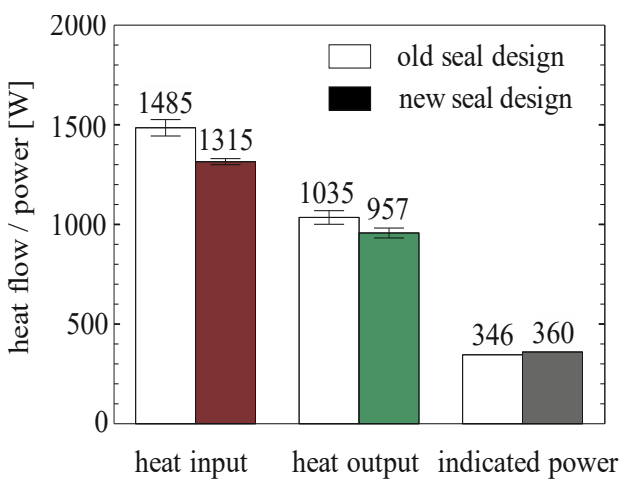

a) Nominal Stirling mode operation

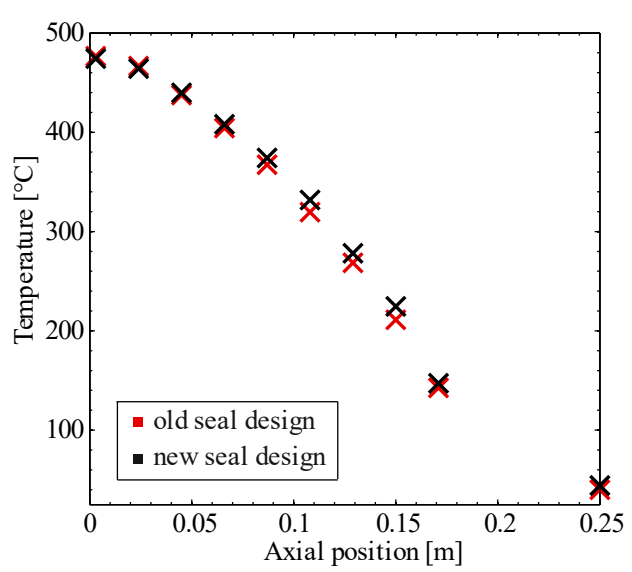

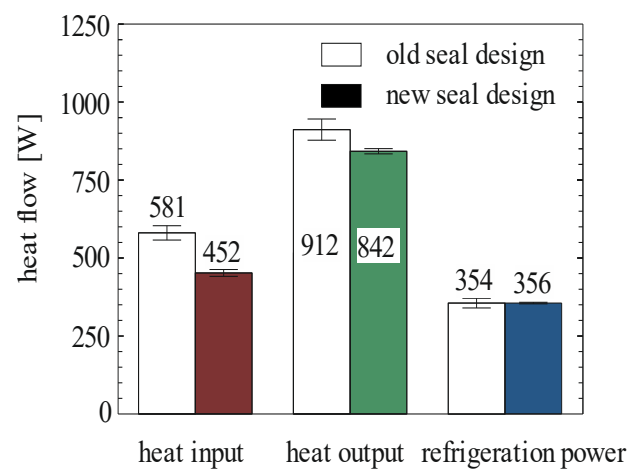

b) Nominal Vuilleumier mode operation

Fig. 10: Externally measured heat flows and indicated power at nominal conditions in Stirling and Vuilleumier mode of the laboratory-scale machine with old and new seal design [25]

Instead, the main reason for the observed positive effects is that the thermal compression by the displacer is actually almost unaffected by the reduction of the seal diameter. This is schematically illustrated in Fig. 11 by a comparison of the volumetric displacement by the seal and the corresponding temperature changes of the gas enclosed in the gap for $r_{h}=1$, $r_{h}=0.5$ and $r_{h}=0$. As a simplification, density changes are ignored, and it is assumed that the local gas temperature equals the arithmetic mean of the adjacent wall temperatures. So, the gas in the gap may be notionally split into two halves featuring the temperature profiles of the cylinder wall and the displacer wall, respectively. Comparing the situation in the TDC and $\mathrm{BDC}$ position of the displacer, it turns out that the actually displaced gas volumes are those bordered by dotted lines, and that only one of these actually undergoes a temperature change. In case of $r_{h}=0$, the latter is not really displaced, but instead, the inner half of the gas in the gap featuring the temperature profile of the displacer wall is assumed to be perfectly regenerated as the displacer moves, which is of course an idealization. In case of $r_{h}=0.5$, the displacement and the temperature change is instead realized by moving this gas quantity (or rather an equivalent quantity of the same size) along the usual flow path from the hot into the warm cylinder volume and vice versa, thus imposing an additional load on the regenerator and causing additional flow losses. In case of $r_{h}=1$, a second gas volume is first heated from $T_{w}$ to $T_{h}$ by the regenerative effect of the cylinder wall and then undergoes the same change of state as described before, further increasing flow losses and regenerator load. 
Summarizing, it turns out that the thermal compression effect by the temperature change of the gas in the gap is almost independent of $r_{h}$. Although the regeneration inside the gap will not be perfect in case of $r_{h}=0$, the dead volume is on the other hand smaller than in case of $r_{h}=1$. So, it is plausible that the pressure amplitude is almost unaffected by the choice of $r_{h}$. Since the component of the overall pressure amplitude that is generated by the thermal compression effect of the displacer is the only one that interacts with the total volume change by the compression piston in Stirling mode or the change of the cold cylinder volume in Vuilleumier mode, it is thus explainable why the indicated power and the refrigeration power are almost unchanged according to Fig. 10. The slight increase of the former may be additionally explained by the reduction of the flow losses, which could be experimentally confirmed [25]. On the other hand, the hot and the warm heat flows are reduced by the smaller volumetric displacement resulting in lower indicated amounts of heat as visualized by the p,V-loop areas in Fig. 9a, and furthermore by decreased regenerator losses because of the smaller mass flow as well as the presumably reduced appendix gap loss, of course. This in turn reduces the thermal load on the adjacent heat exchangers and thus the temperature difference required for the heat transfer. In addition, this enhances the thermal compression effect due to an increased gas temperature difference between the hot and the warm volume.
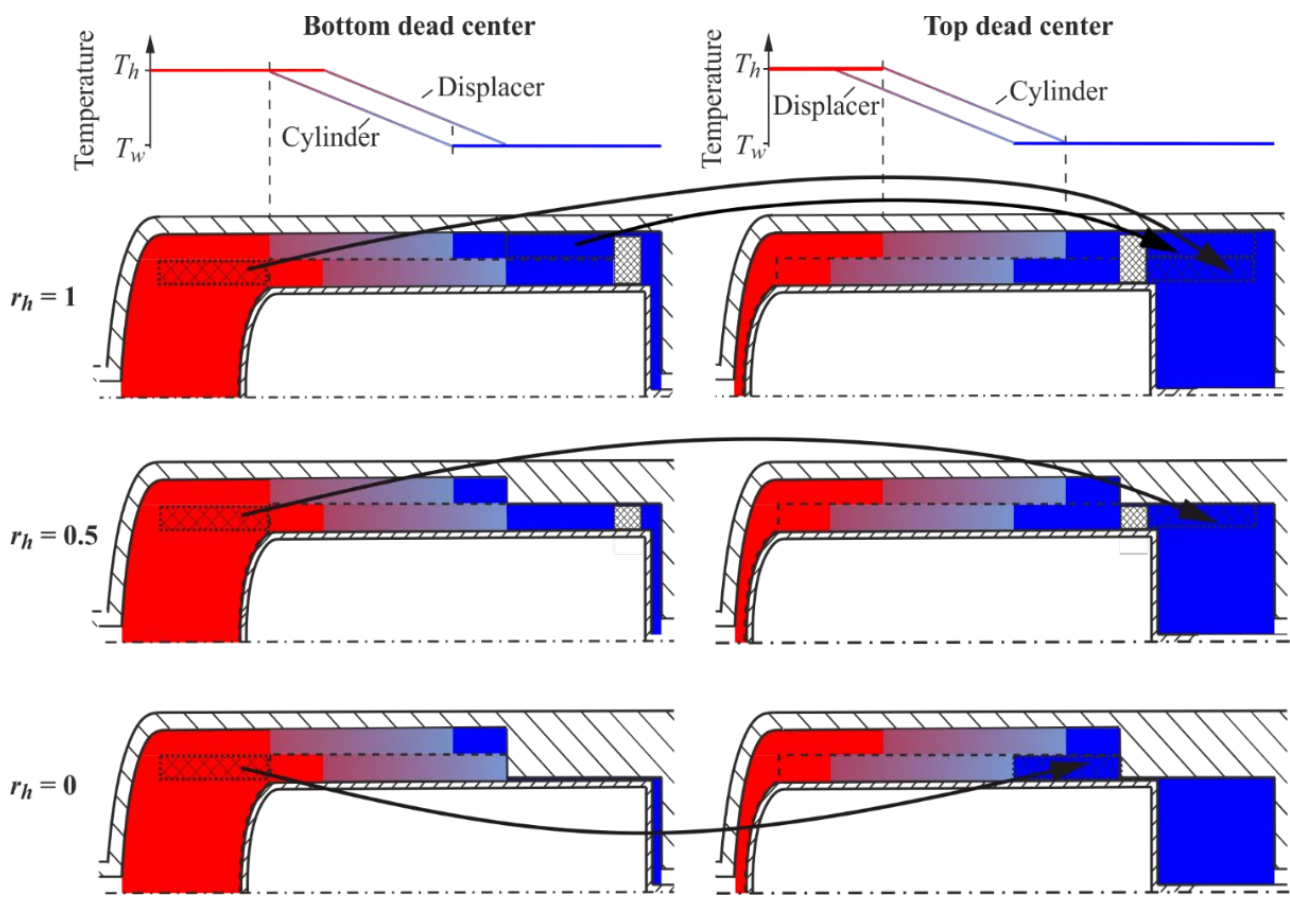

Fig. 11: Comparison of the gas volume displaced by the seal in the appendix gap for $r_{h}=1$, $r_{h}=0.5$ and $r_{h}=0$ with special regard to thermal compression and dead volume effects [25]

Evidently, the observed performance improvements are not only caused by the anticipated reduction of appendix gap loss, which - by the way - could not be directly measured, but only be confirmed indirectly by a comparison of the cyclic gas temperature measurements indicating a reduced mass flow amplitude in the gap and thus a lower enthalpy loss in case of $r_{h}=0.21$. Instead, these improvements are also caused by a series of positive feedback effects involving other cycle components as well as their interaction, which can of course not be described by separate analytical models of the appendix gap and of other components, but only by numerical simulation of the entire cycle including all major loss mechanisms and their interactions, and therefore also including a differential model of the appendix gap. 


\section{Numerical Simulation and Optimization of the Appendix Gap}

As far as known, the first numerical model including a differential simulation of the appendix gap was developed by Andersen [26]. Preliminary results regarding the simulation of the appendix gap loss were previously published by Andersen et al. [11]. Their model is a spatially one-dimensional, so-called third order model, which generally requires a description of any radial heat and momentum transfer by separate, usually empirically fitted correlations. However, hardly any such correlations were available for the description of the radial heat exchange between the gas and the walls in a typical appendix gap, except the correlations obtained by Huang and Berggren [27] on the basis of steady-state heat flow measurements at a P40-R experimental Stirling engine. These yield instantaneous Nusselt numbers for the heat exchange with the cylinder and the displacer wall as empirically fitted functions of the Reynolds number, which is evaluated with the instantaneous flow velocity relative to the cylinder wall in either case. For zero Reynolds numbers, both correlations yield a value of 4 for the Nusselt number, which equivalent to steady-state thermal conduction.

As an alternative, Andersen et al. assumed a parabolic radial gas temperature profile, the coefficients of which can easily be determined from the local wall temperatures and the spatial average gas temperature as obtained from the differential simulation. This is in fact a quasi-stationary approximation of the analytical solution obtained by Pfeiffer and Kühl as displayed in Fig. 3, and therefore it is presumably the better choice in case of laminar flow, whereas the correlations by Huang and Berggren may be a better choice for large gap widths with possibly turbulent flow according to Andersen et al. [11].

To further analyze the appendix gap loss numerically and in particular, to reproduce the aforementioned experimental results, Sauer and Kühl extended an existing third order model for regenerative cycles by a differential simulation of the appendix gap [28]. Because of its modular structure, it may be used to simulate any operating mode of the aforementioned convertible experimental machine, and it features a faster computational speed than the model by Andersen, though at the cost of a simplified handling of the momentum equation.

Concerning the modelling of the radial heat exchange in the gap, the same problem as already described by Andersen et al. was encountered. The results obtained by the parabolic approach and applying the correlations by Huang and Berggren yielded significantly different results. For comparison purposes, plain steady-state thermal conduction was assumed as a third option, once again yielding deviating results as exemplarily illustrated in Fig. 12 for the aforementioned experimental machine. In Addition, the results obtained by the various previously discussed analytical models are also included, demonstrating the discrepancies between all these different approaches. Contrarily to the analytical models yielding a steady increase of the enthalpy loss at rising gap widths, the numerical models predict a maximum at a rather large width. Comparable results were also obtained by Andersen et al. [11], who attributed the decrease of the loss at very large gap widths to an insufficient heat transfer.

Because of these open issues and since neither a satisfactory theoretical nor a sufficient experimental validation of the aforementioned modelling approaches for the heat transfer was available, this issue was investigated in more detail by Sauer and Kühl [29]. To describe the heat transfer, they tentatively applied correlations based on the complex Nusselt number concept, which had previously been derived by Pfeiffer on the basis of the aforementioned unsteady analytical model for the flow and temperature profiles in the gap [30]. Thus, it is possible to account for the phase shift between the heat flux and the temperature difference between the fluid and the wall. However, if the temperature profiles obtained by this model are applied for the modelling of the heat exchange, they should consequently be considered in the description of the axial enthalpy flow in the gap, too. Since both the flow velocity and the radial gas temperature profile show significant variations as exemplarily illustrated in Fig. 3, the axial enthalpy flow in the gap should be evaluated as 

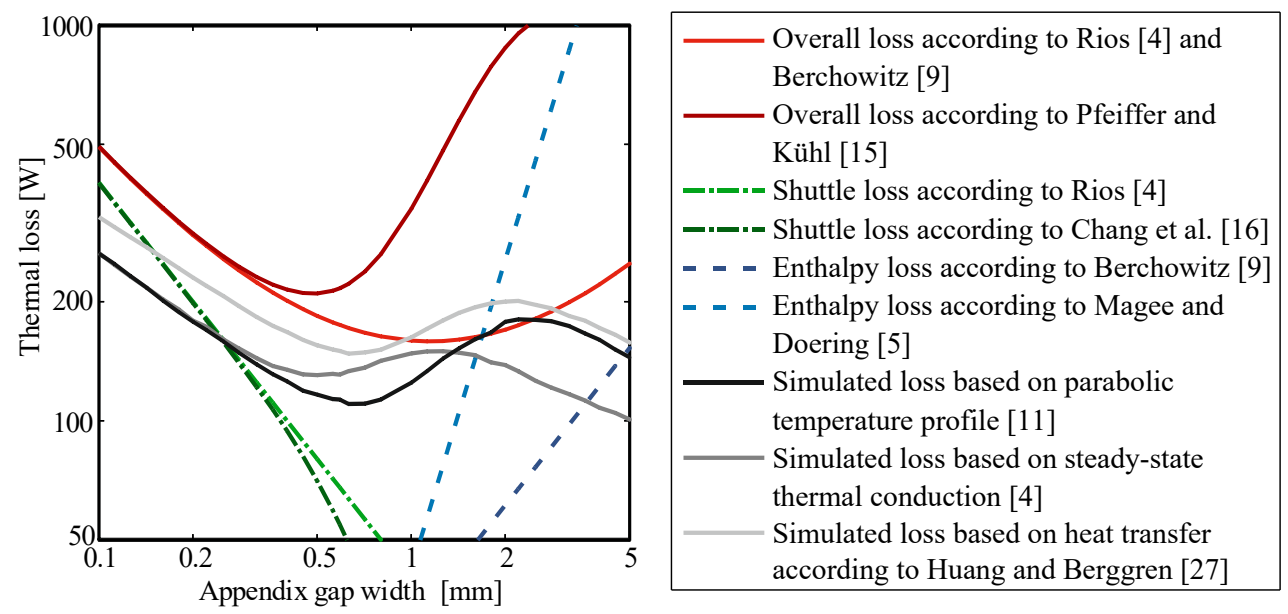

Fig. 12: Dependency of the various loss mechanisms in the appendix gap on the gap width according to the discussed analytical models in comparison to the results of numerical simulations using three different approaches for the modelling of the heat transfer [29]

$$
\dot{H}=\pi d_{C} \rho c_{p} \int_{0}^{h} u(y)\left[T(y)-T_{0}\right] d y
$$

where $T_{0}$ designates an arbitrary zero point temperature of the specific enthalpy. However, in a third order one-dimensional simulation, no information about $u(y)$ and $T(y)$ is available, but only the spatial mean values of the $u_{m}$ and $T_{m}$ instead. Computing the enthalpy flow on the basis of these is only correct in the case of ideal plug flow, i.e., for $u(y)=u_{m}=$ const. and may therefore be an acceptable simplification under highly turbulent flow conditions. However, in all other cases, and particularly in the case of laminar flow, the correct average for the evaluation of the enthalpy flow is the so-called bulk (or "mixing cup") temperature

$$
T_{b}=\frac{1}{u_{m} h} \int_{0}^{h} u(y) T(y) d y
$$

Introducing the mass flow $\dot{m}=\pi d_{C} h \rho u_{m}$, the enthalpy flow may thus be obtained as

$$
\dot{H}=\dot{m} c_{p}\left(T_{b}-T_{0}\right)
$$

Assuming steady-state laminar flow and a heat exchange with a wall at a temperature $T_{w}$, it is possible to quantify the deviation between $T_{b}$ and $T_{m}$ by introducing the ratio

$$
\tau=\frac{T_{b}-T_{0}}{T_{m}-T_{0}}
$$

which turns out to be constant for a given flow situation. In case of laminar flow between parallel plates, which essentially corresponds to the situation in the appendix gap, the result is e.g. $\tau=1.224$ and $\tau=1.214$ for $T_{w}=$ const. and for a constant heat flux, respectively.

However, in unsteady oscillating flow, both the integral in the numerator and $u_{m}$ in the denominator of eq. (13) are periodic functions of the crank angle, and their zero crossings do not coincide due to a phase shift, similarly to the aforementioned unsteady heat exchange with a wall, which could be handled by introducing a constant, complex Nusselt number.

So, the enthalpy flow can only be evaluated by treating both $u(y)$ and $T(y)$ as a sum of complex periodic functions depending on the crank angle by either $e^{i \omega t}$ or $e^{-i \omega t}$. The result is therefore found to consist of three components, one of which is constant, whereas the others are periodic functions of $\omega t$ or $2 \omega t$, respectively. To describe their corresponding phase shifts by complex constants, three different complex temperature ratios $\boldsymbol{\tau}_{\mathbf{1}}, \boldsymbol{\tau}_{\mathbf{2}}$ and $\boldsymbol{\tau}_{\mathbf{3}}$ must be introduced, which are complex functions of $\Gamma, \theta_{u}$ and $P e_{\omega}$ [29]. Implementing these in the third order model, a theoretically founded reference model for the transport phenomena in the gap, which is supported by the experimental results presented in chapter 3 , was created. 
This reference model is difficult to implement and to handle due to its complexity and is therefore not recommendable for practical use. However, it is a useful tool to scrutinize and possibly validate the aforementioned empirical approaches for the heat transfer, which are generally combined with the assumption of ideal plug flow in the evaluation of the enthalpy flow. This was done for the example cases of the GPU-3 engine and the aforementioned experimental machine. Fig. 13 shows the results for the latter as well as those obtained by the analytical models. Evidently, there is a good agreement with the results obtained by the assumption of a parabolic gas temperature profile as suggested by Andersen et al. [11].

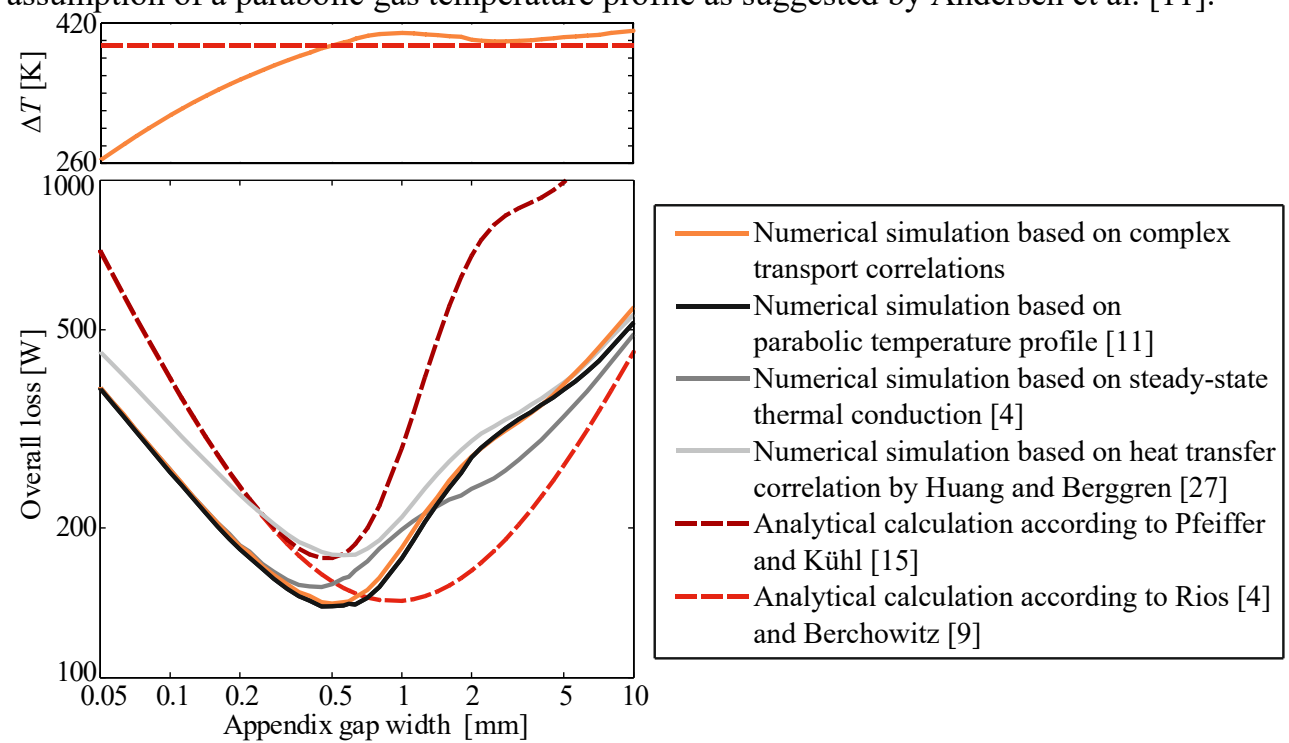

Fig. 13: Results for the dependency of the appendix gap loss on the gap width obtained by numerical simulation using complex transport equations and three empirical approaches in comparison to those obtained by the two specified analytical models [29]

Furthermore, Fig. 13 provides an explanation for the deviating slopes of the curves in the limiting case of vanishing gap widths, which could already be observed in Fig. 12. In this range, the shuttle loss is prevailing, and the heat transfer between the walls can finally be described by steady-state thermal conduction in the gas. In this case, the analytical models yield a slope of -1 in the logarithmic scale due to the dependency on $h^{-1}$, whereas the differential simulations yield a smaller slope. The reason for this deviation is that in the analytical models, the temperature difference $\Delta T=T_{h}-T_{w}$ is assumed as constant, whereas in the numerical model, it is given by the adjacent cylinder wall temperatures. These are dependent on the magnitude of the overall thermal loss in the displacer system, and thus, $\Delta T$ is evidently decreasing at vanishing gap widths due to the increasing shuttle loss.

Finally, the unresolved issue about the maximum of the appendix gap loss at large gap width according to the differential simulation results displayed in Fig. 12 could also be cleared. It is caused by the way how the alleged overall loss was actually computed. In the aforementioned code, this was done by determination of the heat flow that is transferred across the seal section of the displacer system by thermal conduction along the cylinder and the displacer wall, since no other heat transport mechanism exists in this section, if a seal without leakage is assumed. The same method was presumably applied by Andersen et al., who also observed such a maximum. However, a closer analysis of the thermodynamic cycle undergone by the gas in the gap in case of a moving seal (i.e., if we have $r_{h}>0$ ), reveals that the $\mathrm{p}, \mathrm{V}$ work done by the cross section of the seal must be taken into account. In case of an engine, i.e., if the open end of the gap is at an elevated temperature, the gas in the bottom 
end of the gap is expanded because of the decreasing cycle pressure, when the displacer is near its BDC position and the temperature of the gas is low. Near the TDC position of the displacer, the cycle pressure is usually increasing and thus, the gas is compressed at a shifted position and therefore at a more elevated temperature, so that a heat pump effect is generated. The heat flow actually transferred across the seal section is reduced by this effect, which is of course increasing at larger gap widths and thus causes the maximum of the differentially simulated curves in Fig. 12 and the decrease of the alleged overall loss at even higher gap widths. Therefore, the closed-cycle integral of the $\mathrm{p}, \mathrm{V}$ work done by the seal must be added to the aforementioned heat flows across the gap, if the result shall actually reflect the true magnitude of the overall loss, which is in fact the sum of all energy flows conveyed down the temperature gradient along the gap by irreversible mechanisms.

The results of the differential simulations have therefore been corrected accordingly in Fig. 13 and thus show the expected progression to steadily increasing values at larger gap widths. Concerning the optimum gap width, the numerical simulations evidently yield almost the same result as the analytical model by Pfeiffer and Kühl [15], i.e., the shift to lower values compared to the predictions by the earlier analytical models and by eq. (5) is confirmed.

\section{Conclusion and Outlook}

By the development of an enhanced analytical model accounting for the unsteady, oscillating laminar flow in the gap, the theoretical understanding of the loss mechanisms and their dependencies on the various design parameters could be improved, and a new formula for the optimum width could be derived in combination with the recommendation to reduce the bottom gap width ratio $r_{h}$ as far as possible. The predictions of the model concerning the gas temperature profiles in the gap could be largely confirmed by experimental investigations for the center section of the gap at least, whereas major deviations were detected near the ends, since the assumption of fully developed laminar flow is not fulfilled. Nevertheless, this model provides the presumably best approximation of the actual flow situation in the gap that is currently available and was therefore used as a basis for the development of an improved numerical simulation tool accounting for all major losses and their mutual interactions. This tool is now available for the analysis and reproduction of further experimental results, which reveal an overall improvement of the cycle performance by a reduction of $r_{h}$ despite the decrease of the volumetric displacement. Furthermore, it is thus possible to perform numerical optimizations of the entire gap design that may contribute to a better understanding of various parameter effects and to more differentiated design formulas for their optimum values, particularly with regard to $r_{h}$, which need not necessarily be minimized in all cases. Such work has already been performed to some extent and will become available soon [31].

This research was supported by a PhD bursary within the Graduate School of Energy Efficient Production and Logistics in North-Rhine Westphalia, Germany, and funded by the German Research Foundation (Deutsche Forschungsgemeinschaft, DFG), grant numbers KU 755/4-1 and 2. 


\section{References}

1. H.O. McMahon, W.E. Gifford, A new low-temperature gas expansion cycle-Part I, Advances in Cryogenic Engineering 5 (1960), 354-367.

2. G. Walker, Cryocoolers; Part 2: Applications (Plenum Press, New York, London, 1983).

3. P.A. Rios, An analytical and experimental Investigation of the Stirling Engine: Department of Mechanical Engineering. Dissertation, Massachusetts Institute of Technology, 1969.

4. P.A. Rios, An Approximate Solution to the Shuttle Heat-Transfer Losses in a Reciprocating Machine, Journal of Engineering for Gas Turbines and Power 93 (1971), 177-182 https://doi.org/10.1115/1.3445549.

5. F.N. Magee, R.D. Doering, Vuilleumier-Cycle Cryogenic Refrigeration Development, Air Force Flight Dynamics Laboratory (Air Force Flight Dynamics Laboratory, Ohio, 1968).

6. F.J. Zimmerman, R.C. Longsworth, Shuttle Heat Transfer, in: Cryogenic Engineering Conference, Boulder, Colorado, 342-351 (1971).

7. R. Radebaugh, J.E. Zimmerman, Shuttle Heat Transfer in Plastic Displacers at Low Speeds, National Bureau of Standards, Cryogenics Division (National Bureau of Standards, Cryogenics Division, Boulder, Colorado, 1978).

8. D.M. Berchowitz, R.W. Berggren, Appendix Gap Losses in Reciprocating Machines, Mechanical Technology Incorporated (Mechanical Technology Incorporated, Latham, NY, 1981).

9. D.M. Berchowitz, Stirling Cycle Engine Design and Optimization: Faculty of Engineering. Dissertation, University of the Witwatersrand, 1986.

10. I. Urieli, D.M. Berchowitz, Stirling Cycle Engine Analysis (Adam Hilger Ltd, Bristol, 1984).

11. S.K. Andersen, H. Carlsen, P.G. Thomsen, Preliminary results from a numerical study on the appendix gap losses in a Stirling engine, in: International Stirling Association, Proceedings 12th ISEC, Durham UK, 336-347 (2005).

12. I. Geue, J. Pfeiffer, H.-D. Kühl, Laboratory-Scale Stirling-Vuilleumier Hybrid System Part II: Experimental Results, J Propul Power 29 (2013), 812-824 https://doi.org/10.2514/1.B34726.

13. J. Pfeiffer, H.-D. Kühl, Review of Models for Appendix Gap Losses in Stirling Cycle Machines, J Propul Power 30 (2014), 1419-1432 https://doi.org/10.2514/1.B35132.

14. H.-D. Kühl, Wärmetransformationsprozesse ohne Phasenumwandlung thermodynamische Konzeption, Auslegung und Optimierung an Beispielen aus der Wärmepumpen- und Kältetechnik. Habilitationsschrift, Universität Dortmund, Fachbereich Chemietechnik, 2003.

15. J. Pfeiffer, H.-D. Kühl, New Analytical Model for Appendix Gap Losses in Stirling Cycle Machines, Journal of Thermophysics and Heat Transfer 30 (2016), 288-300 https://doi.org/10.2514/1.T4606.

16. H.M. Chang, D.J. Park, S. Jeong, Effect of gap flow on shuttle heat transfer, Cryogenics 40 (2000), 159-166 https://doi.org/10.1016/S0011-2275(00)00020-5.

17. J.H. Baik, H.M. Chang, An Exact Solution for Shuttle Heat-Transfer, Cryogenics 35 (1995), 9-13.

18. J. Pfeiffer, H.-D. Kühl, Optimization of the Appendix Gap Design in Stirling Engines, Journal of Thermophysics and Heat Transfer 30 (2016), 831-842 https://doi.org/10.2514/1.t4729. 
19. L.G. Thieme, Low-Power Baseline Test Results for the GPU 3 Stirling Engine, National Aeronautics and Space Administration (NASA) (NASA; Lewis Research Center, 1979).

20. J. Sauer, H.-D. Kühl, Preliminary Results of Unsteady Gas Temperature Measurements in the Appendix Gap Section of an Experimental Stirling Engine, in: International Stirling Association, Proceedings 17th ISEC, Newcastle upon Tyne, 589-597 (2016).

21. J. Sauer, H.-D. Kühl, Analysis of unsteady gas temperature measurements in the appendix gap of a stirling engine, J Propul Power 34 (2018), 1039-1051 https://doi.org/10.2514/6.2017-4795.

22. I. Geue, J. Pfeiffer, H.-D. Kühl, Laboratory-Scale Stirling-Vuilleumier Hybrid System Part I: Application of Similarity-Based Design, J Propul Power 29 (2013), 800-811 https://doi.org/10.2514/1.B34673.

23. H.-D. Kühl, T. Pfeffer, S. Schulz, C. Walther, High speed gas temperature measurements in a Vuilleumier heat pump and their reproduction by differential computer simulation, in: International Stirling Association, Proceedings 8th ISEC, Ancona, 257-266 (1997).

24. T. Pfeffer, H.-D. Kühl, S. Schulz, C. Walther, Entwicklung und experimentelle Untersuchung neuer Regeneratorkonzepte für regenerative Gaskreisprozesse am Beispiel einer Vuilleumier-Wärmepumpe, Forsch Ingenieurwes 65 (2000), 257-272 https://doi.org/10.1007/s100109900018.

25. J. Sauer, H.-D. Kühl, Experimental Investigation of Displacer Seal Geometry Effects in Stirling Cycle Machines, Energies 12 (2019), 4215 https://doi.org/10.3390/en12214215.

26. S.K. Andersen, Numerical Simulation of Cyclic Thermodynamic Processes: Department of Mechanical Engineering. Dissertation, Technical University of Denmark, 2006.

27. S.C. Huang, R.W. Berggren, Evaluation of Stirling Engine Appendix Gap Losses, in: AIAA, Proc. 21st IECEC, San Diego, CA, 562-568 (1986).

28. J. Sauer, H.-D. Kühl, Numerical model for Stirling cycle machines including a differential simulation of the appendix gap, Applied Thermal Engineering 111 (2017), 819-833 https://doi.org/10.1016/j.applthermaleng.2016.09.176.

29. J. Sauer, H.-D. Kühl, Theoretically and experimentally founded simulation of the appendix gap in regenerative machines, Applied Thermal Engineering 166 (2020), 114530 https://doi.org/10.1016/j.applthermaleng.2019.114530.

30. J. Pfeiffer, Unsteady Analytical Model for Appendix Gap Losses in Stirling Cycle Machines, Dissertation, Lehrstuhl für Thermodynamik, TU Dortmund (Dr. Hut, München, 2016).

31. J. Sauer, H.-D. Kühl, Performance Improvements in Stirling Cycle Machines by a Modified Appendix Gap Geometry, Int. J. Energy Res. (submitted). 